\title{
Associations among Task Self-Efficacy, Physical Activity and Subjective Wellbeing
}

Abigail M. Nehrkorn

Follow this and additional works at: https://researchrepository.wvu.edu/etd

\section{Recommended Citation}

Nehrkorn, Abigail M., "Associations among Task Self-Efficacy, Physical Activity and Subjective Wellbeing" (2016). Graduate Theses, Dissertations, and Problem Reports. 6303.

https://researchrepository.wvu.edu/etd/6303

This Thesis is protected by copyright and/or related rights. It has been brought to you by the The Research Repository @ WVU with permission from the rights-holder(s). You are free to use this Thesis in any way that is permitted by the copyright and related rights legislation that applies to your use. For other uses you must obtain permission from the rights-holder(s) directly, unless additional rights are indicated by a Creative Commons license in the record and/ or on the work itself. This Thesis has been accepted for inclusion in WVU Graduate Theses, Dissertations, and Problem Reports collection by an authorized administrator of The Research Repository @ WVU. For more information, please contact researchrepository@mail.wvu.edu. 
Associations among Task Self-Efficacy, Physical Activity and Subjective Wellbeing

\author{
Abigail M. Nehrkorn \\ Thesis submitted to the \\ Eberly College of Arts and Sciences \\ at West Virginia University \\ in partial fulfillment of the requirements \\ for the degree of
}

\author{
Master of Science \\ in \\ Psychology
}

\author{
Julie Hicks Patrick, Ph.D., Chair \\ Constance Toffle, Ph.D. \\ Elizabeth Kyonka, Ph.D. \\ Department of Psychology
}
Morgantown, West Virginia 2016

Keywords: physical activity, self-efficacy, wellbeing, adults Copyright 2016 Abigail M. Nehrkorn 


\begin{abstract}
Associations among Task Self-Efficacy, Physical Activity and Subjective Wellbeing
\end{abstract}

Abigail M. Nehrkorn

The physical and psychological health benefits resulting from physical activity engagement have been documented in previous literature, including the connection between physical activity and subjective wellbeing (SWB). Associations have also been found between task-related self-efficacy and physical activity, connecting these concepts to Bandura's Social Cognitive Theory (SCT; Bandura, 1982, 1997), such that retaining a higher belief in one's physical activity abilities has been associated with successful physical activity engagement. Previous literature has documented these separate associations, but not as much focus has been placed on examining task self-efficacy, physical activity, and SWB in one model, especially during the time of physical activity adoption. Utilizing data from 58 adults, we conducted a path analysis to examine autoregressive and cross-lagged paths that encompassed 4 waves of data and 3 weeks of time. Significant autoregressive trends were observed for task self-efficacy, physical activity, and SWB, such that these variables increased over time, but the included cross-lagged paths were not found to be significant, indicating a lack of a relationship between task selfefficacy and physical activity, between physical activity and SWB, and between task selfefficacy and SWB. Significant age effects did not emerge, indicating similar scores in task selfefficacy, physical activity, and SWB across younger, middle-aged, and older adults. Similarities and differences between our study and the previous literature are discussed, along with proposing crucial aspects to consider in future studies and interventions on this topic. 


\section{Table of Contents}

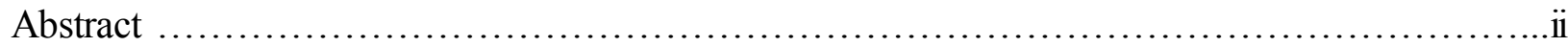

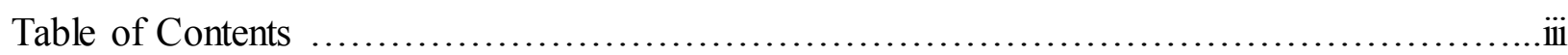

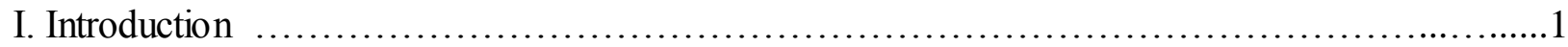

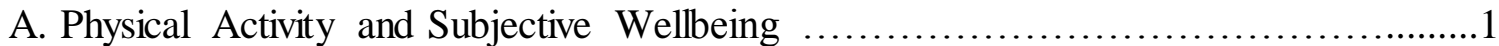

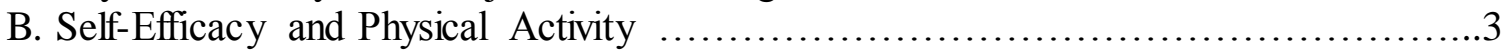

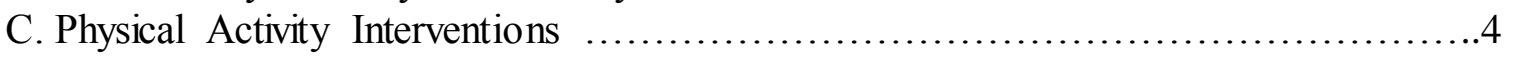

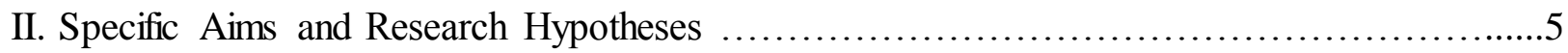

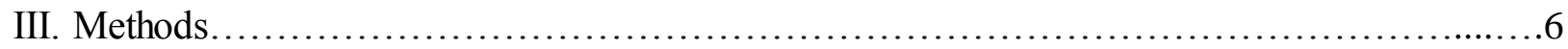

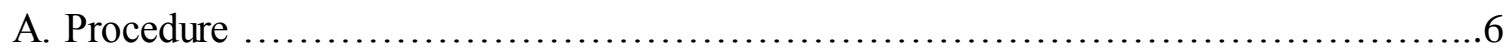

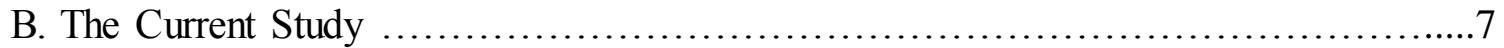

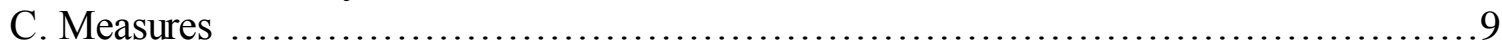

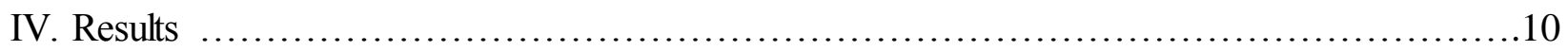

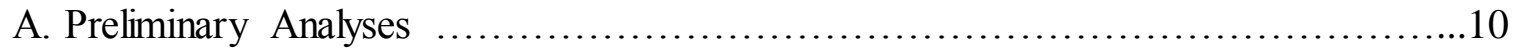

B. Autoregressive Paths .............................................................

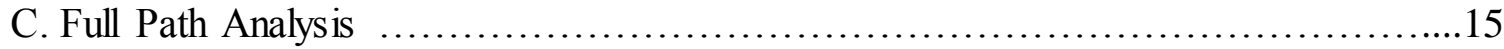

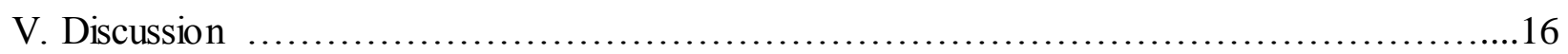

A. Hypothesis 1: Autoregressive Paths across Task Self-Efficacy, Physical Activity and SWB ........................................................................ 16

B. Hypothesis 2: Task Self-Efficacy and Physical Activity .........................17

C. Hypothesis 3: Physical Activity and SWB ..................................... 18

D. Hypothesis 4: Age ...................................................... 19

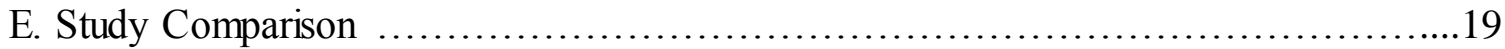

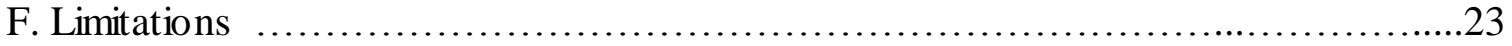

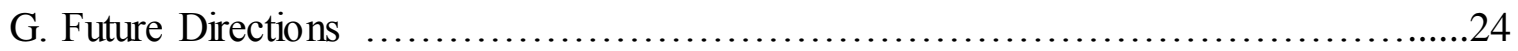

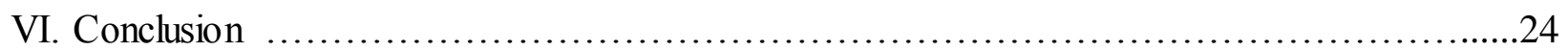

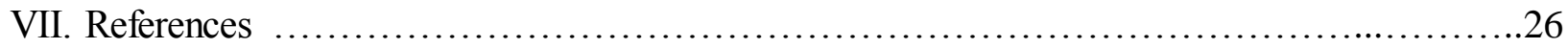

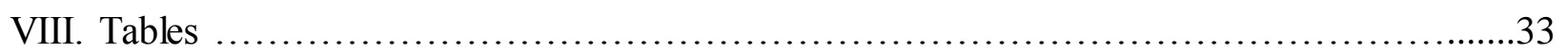

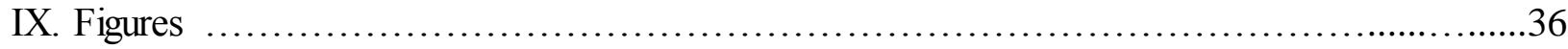

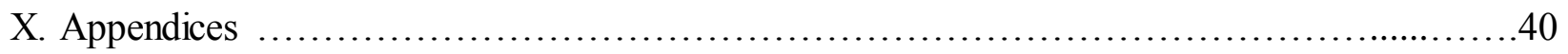


Associations among Task Self-Efficacy, Physical Activity and Subjective Wellbeing The benefits of participating in moderate to vigorous physical activity have been linked to enhanced physical health outcomes, decreased risks of developing chronic diseases or illnesses, an increased level of personal wellness, and higher quality of life (Baranowski et al., 1997; Barwais, Cuddihy, \& Tomson, 2013; English, Manns, Tucak, \& Bernhardt, 2013; Gross, 2013; Phelan, Anderson, Lacroix, \& Larson, 2004; Schoenborn, Adams, and Peregoy, 2013). Although engaging in moderate to vigorous physical activity is important for present health, many adults are not meeting these requirements. Schoenborn et al. (2013) report that fewer than half of adults meet current physical activity recommendations, with only $46.1 \%$ of adults taking part in 150 minutes of moderate physical activity or 75 minutes of vigorous activity each week. Along with other health-risk factors, and due to the current population not reaching these recommendations and becoming increasingly sedentary, the prevalence of obesity has been increasing in the past decade (Anderson, Wojcik, Winett, \& Williams, 2006). Because physical activity is a highly modifiable lifestyle factor, it may play an important role in helping to improve the health of adults (Friedenreich \& Orenstein, 2002).

\section{Physical Activity and Subjective Wellbeing}

Failing to engage in adequate physical activity is harmful not only for physical health, but it also has a negative effect on subjective wellbeing. Subjective wellbeing (SWB) is comprised of life satisfaction, positive affect, and negative affect (Diener, 2000; Diener, Oishi, \& Lucas, 2003). A high sense of SWB is desirable for people across cultures and generations (Emmons \& McCullough, 2003).

Physical activity and physical health are correlates of SWB (Dolan, Peasgood, \& White, 2008; Fox, 1999; McAuley, Konopack, Motl, Morris, Doerksen, \& Rosengren, 2006). Older adults with more acute illnesses, more chronic health conditions, and more physical limitations 
(e.g., knee problems) report lower SWB (Garatachea, Molinero, Martínez-García, JiménezJiménez, González-Gallego \& Márquez, 2009; Mullen, McAuley, Satariano, Kealey, \& Prohaska, 2012; Rejeski \& Mihalko, 2001). However, depression levels are lower among older adults who exercise (Singh, Stavrinos, Scarbek, Galambos, Liber, \& Singh, 2005). Thus, there is a recognized relation between SWB and physical activity.

The relation between physical activity and SWB may be causal. Physical activity can potentially provide psychological benefits by leading to more positive emotions and more positive beliefs about the self (Fox, 1999). Additionally, sustained physical activity can lead to a reduction in anxiety, improved mood, enhanced health quality, improved sleep quality, reduced reactivity to stressors, and improved cognitive functioning (Arent, Landers, \& Etnier, 2000; Fox, Stathi, \& McKenna, 2007; Rejeski \& Mihaiko, 2001; Schoenborn et al., 2013). Chaddock, Voss, \& Kramer (2012) explain that physically active older adults perform higher on a variety of cognitive tasks and are at a lower risk for developing dementia, Atzheimer's disease, or other cognitive impairments. Thus, among older adults, the structure, function, and health of the brain may benefit from physical activity. So, by living a sedentary lifestyle, a variety of mental, physical, cognitive, and emotional health aspects can be affected, some of which can lead to a lower SWB (Chaddock et al., 2012; Diener et al., 2003).

It has been noted that a lack of exercise may affect wellbeing differently across people due to the fact that some may be or have been more physically active or have more exercise experiences than others (Netz, Wu, Becke, \& Tenenbaum, 2005). Netz and colleagues (2005) reported that the wellbeing of past athletes could experience a harsher effect in the instance of a lack of exercise. Other factors, such as marital status, temperament, personality, culture, 
socioeconomic status, social support, and societal factors also can affect SWB (Diener, 2000; Dolan et al., 2008).

\section{Self-Efficacy and Physical Activity}

Self-efficacy is also associated with physical activity engagement. As discussed in Social Cognitive Theory (SCT), self-efficacy plays a central role in the process of learning, creating goals, and successfully reaching some target experience (Bandura, 1997, 1998). Self-efficacy can be defined as one's confidence in one's own agency (Bandura, 1977). It can be differentiated into various types, consisting of global self-efficacy, domain-specific self-efficacy, and taskrelated self-efficacy. Task-related self-efficacy corresponds to examining task demands and its relation to a successful performance (Bandura, 2006). Domain-specific self-efficacy refers to focusing on the measure of efficacy in a particular area of interest, such as academic subjects (Bandura, 2006). Global self-efficacy refers to attitudes and competence in abilities towards a broad range of tasks, causing it to be a much more general type of self-efficacy (Luszczynska, Scholz, \& Schwarzer, 2005; Pajares, 1997).

Task-related physical activity self-efficacy is the belief that one can successfully accomplish some physical activity or exercise, when in regards to a physical activity task (Bandura, 1997; Olander, Fletcher, Williams, Atkinson, Turner, \& French, 2013). Seghers, Van Hoecke, Schotte, and Opdenaker (2014) examined task-related self-efficacy among 239 participants (ages 18 to 65 years) during a 12-week physical activity intervention program in which participants were either part of the standard-intervention group or the extra-intervention group, which included additional coaching on increasing self-efficacy. Seghers and colleagues (2014) found that by including the self-efficacy coaching, health coaching resulted in an increase in both task- and domain-specific efficacy. Importantly, there was also a decrease in sedentary 
behaviors, an increase in SWB, and a decrease in body mass index (BMI; Seghers et al., 2014). Whether self-efficacy is a necessary precursor to engaging in physical activity, a consequence of engaging in physical activity, or simply changes in parallel, remains to be determined (Ayotte, Margrett, \& Patrick, 2013; McAuley et al., 2007; Mullen et al., 2012). However, if the person feels confident about their ability to complete the activity, they will be more likely to engage in the activity and be successful (Resnick, Palmer, Jenkins, \& Spellbring, 2000). In sum, selfefficacy significantly predicts maintenance of physical activity for the near future, for the longer maintenance stage, and contributes to SWB (Ayotte et al., 2013; Codina et al., 2013; McAuley, 1993; McAuley, Jerome, Elavsky, Marquez, \& Ramsey, 2003; Seghers et al., 2014).

\section{Physical Activity Interventions}

Many people initiate physical activity and other new habits, but fail to maintain that behavior change across time (McAuley, Courneya, Rudolph, \& Lox, 1994). In connection with physical activity, SWB, and self-efficacy, age may act as a moderator that affects these variables differently (Anderson et al., 2006; Netz et al., 2005; Rejeski \& Mihalko, 2001). In regards to physical activity, older adults are engaging in the least amount of exercise and spending the most time in a sedentary lifestyle (Schoenborn et al., 2013; Withall, Stathi, Davis, Coulson, Thompson, \& Fox, 2014). By the age of 35 years and further into middle and older adulthood, there is a general decline of the major biological systems of the lungs, muscles, and heart (Renner, Spivak, Kwon, \& Schwarzer, 2007; Rybash, Roodin, \& Hoyer, 1986). To combat this sedentary lifestyle, research has shown that developing physical activity routines and maintaining them is crucial in childhood and younger adulthood (Marcus et al., 2000). Caspersen, Pereira, \& Curran (2000) note how adults from 18 to 29 years often become detached from their previous exercise routines, eliminating the maintenance component. So, to stray from 
the lack of physical activity in middle and later adulthood and to be proactive against major systems declining, younger adults should consider creating and maintaining a consistent physical activity level and routine.

Sex, along with age, can influence physical activity levels, self-efficacy, and SWB of the person. Focusing on men and women, women report lower physical activity levels than do men (Caspersen et al., 2000; Lutter, 1995; Segar, Jayaratne, Hanlon, \& Richardson, 2002; Verhoef, Love, \& Rose, 1992). As is connected with age, as women grow older, their levels of physical inactivity and sedentary behavior increase (Caspersen et al., 2000; Segar et al., 2002; Withall et al., 2014). Mediators to consider for middle- and older-aged women include the lack of sports participation available when they were younger, as well as currently, along with responsibilities to work and family (Lutter, 1994; Segar et al., 2002). Due to these many mediators, Segar and colleagues (2002) have expressed the importance for women to address their responsibilities and commitments, such as being a stay-at-home mom or businesswoman, and to work around these barriers to increase their physical activity levels.

\section{Specific Aims and Research Hypotheses}

By offering health coaching programs that include a self-efficacy piece, adults may be more likely to increase activity, adhere to physical activity guidelines, and maintain this activity throughout the course of their life (Baranowski et al., 1997; Butterworth, Linden, \& McClay, 2007). The purpose of this study was to examine the effects of task-related self-efficacy on changing physical activity and SWB throughout the initial period of adopting a new physical activity behavior. It was hypothesized that as a function of self-monitoring and health coaching, task self-efficacy, physical activity, and SWB increased over time (Hypothesis 1). Guided by SCT (Bandura, 1998), it was expected that self-efficacy and physical activity were related over 
time (See Figure 1). That is, initial task self-efficacy was expected to be associated with Time 2 physical activity (Hypothesis 2). Further, as adults became more physically active, it was hypothesized that SWB (i.e. increased life satisfaction) increased (Hypothesis 3). It was hypothesized that age did not influence the model, but rather all ages had similar task selfefficacy, physical activity, and SWB (Hypothesis 4).

\section{Methods}

\section{Procedure}

Data for the proposed study were provided by adults who participated in the Age and Behavioral Coaching across Domains study (Patrick \& Gentzler, PIs, Institute on Coaching). The parent study, which included a minimum of 96 adults, was designed to include a 12-week protocol.

The purpose of the parent study was to have health coaches work with participants, who had listed a physical activity goal that they wished to work on. Participants were required to first complete a prescreen before being invited to participate in the study. It was in the prescreen that participants indicated a personally relevant physical activity goal. See Appendix D for information concerning the prescreen. After completing the prescreen and being invited to partake in the study, participants were assigned to be in one condition, with there being three possible conditions: a group that utilized accelerometers, a group that utilized social support, and a group that did not have either accelerometers or social support. Preliminary analyses examined condition effect, but there were not any significant associations between condition and the outcome variables, eliminating it from subsequent analyses. Figure 1 details the process of inclusion into the study. 
The first part of the parent study began after having completed the prescreen and being assigned to a condition. The initial part of the study included a three-week protocol, during which participants would meet in-person with their health coach. During the three weeks of inperson meeting, measures were utilized that assessed other areas besides physical activity and physical health, including cognition, emotions, spirituality, and other health-related measures. A wide range of measures were included due to the fact that a change in one domain of a person's life (i.e., physical activity engagement) can initiate subsequent changes in other areas of their life (i.e., cognitions, emotions, etc.). The remaining 9 weeks of the study did not include any inperson meetings, but participants completed online surveys, that assessed these various domains, as well.

The health coaches assigned to participants were trained members of the Healthy Aging Lab. As weekly concrete strategies are utilized to aid the participant in reaching their goal (i.e., walking one mile two times a week), the health coach worked with the participants to break down their goal into manageable, concrete strategies. Due to its focus on health education and promotion, health coaching has been found to be successful in promoting healthy attitudes, along with transferring information to participants concerning skills to manage their health (Butterworth et al., 2007; Palmer, Tubbs, \& Whybrow, 2003).

\section{The Current Study}

To examine the associations among task self-efficacy, physical activity, and SWB, data from 37 younger adults (ages 18-39 years) and 20 middle-aged and older adults (ages 40-72 years) were used. Data from Week 0 (Baseline), Week 1, Week 2, and Week 3 were utilized for physical activity and SWB. Task self-efficacy was assessed at Week 0 and Week 3 data. These weeks, encompassing the first part of the parent study, were chosen as they contain measures that 
question the participant on their task self-efficacy, physical activity, and SWB. All four weekly contacts were conducted in-person between the health coach and participant. By including consecutive weeks of measurement from the start of the study, the focus was on activity initiation and how physical activity, task self-efficacy, and SWB progressed from the initial meeting.

Four participants dropped out from the study at Week 0 or Week 1 and due to the amount of missing data from these cases, they were excluded from analyses. Outliers were assessed and it was found that across the 4 waves, there were 14 outliers. Due to the smaller sample size, analyses were utilized to bring the outliers closer to the rest of the sample. Outliers were windsorized, meaning they received new scores that were 5\% away from the highest or lowest non-outlier value (Ghosh \& Vogt, 2012). This is a common practice to avoid throwing out data and to lessen the severity of a variable's skew (Ghosh \& Vogt, 2012). A total sample size of $\mathrm{N}=$ 58 was then utilized in the study. A power analysis was implemented in $G^{*}$ Power for a repeated measures ANOVA analysis for within-subjects, suggesting that a $\mathrm{N}=27$ would provide sufficient power $(>.85)$ to detect a medium-sized effect $(f=.25)$ at $\mathrm{p}<.05$. Thus, the current study's total sample size was deemed acceptable to ensure sufficient power.

A path analysis was conducted in AMOS 21.0 (Arbuckle, 2012), including the variables of task self-efficacy, physical activity, and SWB. Data that was missing, but not in excess was imputed using the AMOS regression imputation process (Arbuckle, 2012). Autoregressive analyses were performed to examine the variables over the 4 waves. Cross-lagged analyses were also conducted in order to assess any associations across variables over the 4 waves. Multiple repeated measures ANOVA analyses were performed to explore associations of each age group with physical activity and SWB, along with associations between the variables and sex, 
education, and race. A paired t-test was also employed to explore further associations for task self-efficacy.

\section{Measures}

Means and standard deviations are presented in Table 1. Pearson correlations are represented in Table 2. Each of the major constructs utilized is discussed below.

\section{Demographics.}

Participants ranged in age from 18 to 72 years of age $(M$ age $=35 ; S D=16.8)$ and included 37 younger $(M$ age $=23.4 ; S D=5.31)$ and 20 middle aged and older adults $(M$ age $=$ $56.3 ; S D=6.67)$. Almost three-quarters $(73.6 \%)$ of the participants were female. The vast majority of the sample identified as Caucasian (91\%) and all adults had completed high school.

\section{Task Self-Efficacy.}

The Task-Related Self-Efficacy Scale (Bray \& Cowan, 2004) consists of nine items in which adults report their confidence (0 to 100\%) that they could exercise continuously for different amounts of time (5 minutes to 45 minutes). As participants give higher percentages, this indicates a higher level of task self-efficacy towards their physical activity goal. A sample mean of $84.28(S D=18.25, \alpha=.92)$ was obtained for Week 0 and a sample mean of $87.42(S D=$ 16.95) was obtained for Week 3. The internal reliability for this scale has been high in past research $(\alpha=.87$ - .96; Ayotte et al., 2013; Bray \& Cowan, 2004).

\section{Subjective Wellbeing.}

The Satisfaction with Life Scale (Diener, Emmons, Larsen, \& Griffin, 1985) includes five Likert-type statements to which participants indicate their agreement or disagreement (1=Strongly Disagree to 7=Strongly Agree). The scale is scored such that higher scores reflect higher levels of SWB. A sample mean of $24.82(S D=6.02, \alpha=.92)$ was obtained for Week 0 , a 
sample mean of $25.76(S D=5.9)$ was obtained for Week 1 , a sample mean of $27.17(S D=5.47)$ was obtained for Week 2 , and a sample mean of $27.09(S D=5.91)$ was obtained for Week 3 . Internal consistency is generally good $(\alpha=.87$; Diener et al, 1985), with convergent validity data of single-item SWB also providing acceptable reliability $(\alpha=.40-.66$; Andrews \& Withey, 1976).

\section{Physical Activity.}

The Godin Leisure Time Exercise Questionnaire (Godin \& Shephard, 1997) will be utilized, as a subjective measure of physical activity. The questions in this measure ask how often, in groupings of 15 minutes, the participant has engaged in strenuous, moderate, and mild physical activity. Along with each level of physical activity, examples of exercise are included, providing the participant with ideas of their past week exercise, as well as ideas for future exercise. Scores are calculated using metabolic equivalent (MET) values for strenuous, moderate, and mild physical activities, which are multiplied by time spent engaging in corresponding strenuous, moderate, and mild activity. The equation utilized to result in a weekly physical activity score is: $(9 \times$ Strenuous $)+(5 \times$ Moderate $)+(3 \times$ Mild $)$. A sample mean of $50.24(S D=36.4, \alpha=.65)$ was maintained for Week 0 , a sample mean of $92.71(\mathrm{SD}=65.98)$ was obtained for Week 1, a sample mean of $93.69(S D=56.44)$ was obtained for Week 2 , and a sample mean of $95.34(S D=52.23)$ was obtained for Week 3. The Cronbach's alpha was generated by including all 3 items into one scale. The reliability for this questionnaire has been acceptable in past research $(\alpha=.60$; Okun, Karoly, \& Lutz, 2002).

\section{Results}

\section{Preliminary Analyses}

Pearson correlations were first analyzed for an initial understanding of associations. As 
shown in Table 2, age was not significantly associated with task self-efficacy at Week $0(r=-$ $.22)$ or Week $3(r=-.12)$. As was previously discussed, task self-efficacy was only measured at Week 0 and Week 3, which means that no data for task self-efficacy will be reported for Week 1 or Week 2. Age was also not significantly associated with Week 0 physical activity $(r=.05)$, Week 1 physical activity $(r=-.01)$, Week 2 physical activity $(r=.01)$, or Week 3 physical activity $(r=-.10)$. Similarly, age was not significantly correlated with Week 0 SWB $(r=.09)$, Week 1 SWB $(r=.16)$, Week 2 SWB $(r=.02)$, or Week 3 SWB $(r=.18)$. Concerning education, there was not a significant association with task self-efficacy at Week $0(r=.14)$ or at Week $3(r$ $=.06)$. For physical activity, there was not a significant association with education at Week $0(r$ $=.34)$, Week $1(r=.11)$, or Week $3(r=.35)$, but there was a significant association at Week $2(r$ $=.43)$. However, this single time point resulting in a significant association seems spurious due to the lack of significant associations among the other time points for physical activity. In regard to SWB, there was not a significant association with education at Week $0(r=.003)$, Week $1(r=$ -.09), Week $2(r=.15)$, or Week $3(r=.27)$. Sex was not significantly associated with task selfefficacy at Week $0(r=.045)$ or Week $3(r=.17)$. Sex was not significantly associated with Week 0 physical activity $(r=.17)$, Week 1 physical activity $(r=.15)$, Week 2 physical activity $(r$ $=.20)$, or Week 3 physical activity $(r=.29)$. SWB was not significantly associated with sex at Week $0(r=-.12)$, Week $1(r=-.12)$, Week $2(r=.06)$, or Week $3(r=-.08)$. See Table 2 for the bivariate correlations across the three variables, along with potential covariates.

Looking further at correlations, variables showed positive correlations across waves. As is detailed in Table 2, task self-efficacy at Week 0 was significantly correlated with Week 3 task self-efficacy $(r=.85)$. Furthermore, SWB at Week 0 was significantly associated with SWB at Week $1(r=.91)$, which was significantly associated with Week $2(r=.81)$, which was 
significantly correlated with Week $3(r=.68)$. For physical activity, Week 0 was significantly associated with Week $1(r=.31)$, which was not significantly associated with Week $2(r=.25)$, but Week 2 was significantly associated with Week 3 physical activity $(r=.69)$. There were not significant associations between variables, however, as is shown in Table 2 .

A paired t-test was performed for task self-efficacy and repeated measures ANOVA analyses were conducted for SWB and physical activity to elaborate on associations found from the Pearson correlations. Performing these additional analyses allowed us to further analyze the relationship of change by comparing means across each time of measurement. Additionally, repeated measures ANOVA analyses can lend information concerning whether covariates should be included into the path analysis.

Due to only having two waves of data for task self-efficacy, a paired t-test was conducted in order to see if changes had occurred since Week 0 . There was a significant increase from Week 0 task self-efficacy $(M=84.28, S D=18.25)$ to Week 3 task self-efficacy $(M=87.42, S D$ $=16.95), t=-2.21, p<.05$. This indicates that as people progressed from the first time point, they scored higher on task self-efficacy.

A repeated measures ANOVA analysis for SWB was conducted, studying the 4 waves with and without covariates added into the model. Mean SWB showed a significant change over the waves when a Sphericity Assumed correction was utilized, $F(3,156)=5.84, p<.01, \eta^{2}=$ .10. See Table 3 for results of the repeated measures ANOVA analyses. Pairwise comparisons were then examined to analyze change. Week $0(M=24.82, S D=6.02)$ had a significantly lower SWB score, $p<.05$, than Week $1(M=25.76, S D=5.9)$, a significantly lower SWB score, $p<$ .001 , than Week $2(M=27.17, S D=5.47)$, and a significantly lower SWB score, $p<.05$, than Week $3(M=27.09, S D=5.91)$. SWB scores at Week 1 were not significantly different from 
Week 2. A repeated measures ANCOVA was performed for SWB, but there was not a significant Time*Age within-subjects effect using the Sphericity Assumed correction, $F(3,150)$ $=1.30, p=.28$, partial $\eta^{2}=.03$, which supports hypothesis 4 . When sex was applied to the SWB model as a covariate and utilized the same correction, it was also non-significant, $F(3,150)=$ 2.31, $p=.08$, partial $\eta^{2}=.04$. Education, as a covariate in the model, was also non-significant with the same correction, $F(3,60)=1.40, p=.25$, partial $\eta^{2}=.07$. Lastly, race was included as a covariate of the SWB model and utilizing the same Sphericity Assumed correction, it was nonsignificant, $F(3,150)=.30, p=.83$, partial $\eta^{2}=.01$. These results imply that age group, sex, education level, nor race had an effect on SWB scores.

A repeated measures ANOVA analysis for physical activity was performed, which is followed by a description of the associations with the included covariates. The mean physical activity score significantly changed across the waves when utilizing the Sphericity Assumed correction, $F(3,156)=10.79, p<.001$, partial $\eta^{2}=.172$. See Table 3 for results of the repeated measures ANOVA analyses. Further analyzing the trajectory of physical activity change, pairwise comparisons were utilized. Week 0 physical activity $(M=50.24, S D=36.4)$ was significantly lower, $p<.001$, than physical activity at Week $1(M=92.71, S D=65.98)$, was also significantly lower, $p<.001$, than Week 2 physical activity $(M=93.69, S D=56.44)$, and was also significantly lower, $p<.001$, than physical activity at Week $3(M=95.34, S D=52.23)$. However, none of the other waves significantly differed from each other. Age was not a significant covariate to the model when using the Sphericity Assumed correction for withinsubjects tests, $F(3,150)=.177, p=.91$, partial $\eta^{2}=.004$, which supports hypothesis 4 . Sex was a non-significant covariate of the same model, $F(3,150)=.03, p=.995$, partial $\eta^{2}=.001$. The Sphericity Assumed correction for within-subjects effects showed education to be a non- 
significant covariate of time, $F(3,150)=.41, p=.75$, partial $\eta^{2}=.02$. Lastly, race was included as a covariate in the model with the same correction and was non-significant, $F(3,150)=1.67, p$ $=.18$, partial $\eta^{2}=.03$. None of the included covariates had an effect on physical activity scores across the waves either. Due to age, sex, education, and race being non-significant, they were not included in the path analysis

\section{Autoregressive Paths}

The complete path analysis model shown in Figure 1 was tested in AMOS. Regression imputation in AMOS was conducted for the path analysis (Arbuckle, 2012), although the original dataset was utilized for the preliminary analyses. The overall model was assessed as a means to test hypotheses 2 and 3, but we also focused on specific autoregressive pathways within the model to test hypothesis 1 . Autoregressive trends were first analyzed to understand how taskself-efficacy, physical activity, and SWB progressed over the waves. These trends, highlighting hypothesis 1, are shown in Figure 2. To assess model fit and autoregressive trends, a path analysis that only contained autoregressive trends was first tested. For this autoregressive model,

the chi-square statistic was significant, $\chi^{2}=67.34, d f=36, p<.01$, Week 3 task self-efficacy $\mathrm{R}^{2}$ $=.731$, Week 3 SWB $\mathrm{R}^{2}=.464$, and Week 3 physical activity $\mathrm{R}^{2}=.482$. Other indices of model fit were mixed, $\mathrm{CMIN} / d f=1.87, \mathrm{CFI}=0.91, \mathrm{RMSEA}=0.12$. These indices only partially supported a well-fitting model, as the CMIN/df statistic was the only adequate value. The CFI and RMSEA values supported a poor-fitting model. The model fit is a noted limitation.

There was a significant path from Week 0 to Week 3 for task self-efficacy, $\mathrm{b}=.791, p<$ .001. For physical activity, there was a significant path from Week 0 to Week $1, \mathrm{~b}=.549, p<$ .05 , a non-significant path from Week 1 to Week $2, \mathrm{~b}=.212, p=.05$, and a significant path from Week 2 to Week $3, \mathrm{~b}=.663, p<.001$. For SWB, there was a significant path from Week 0 to 
Week $1, \mathrm{~b}=.874, p<.001$, a significant path from Week 1 to Week $2, \mathrm{~b}=.757, p<.001$, and $\mathrm{a}$ significant path from Week 2 to Week $3, \mathrm{~b}=.726, p<.001$. As was hypothesized, there were strong direct relations for each of the variables across the waves, except for physical activity during the two middle waves.

\section{Full Path Analysis}

Following the analysis of the autoregressive paths, cross-lagged paths were then included into the model. For the whole path analysis with autoregressive and cross-lagged pathways, the chi-square statistic was significant, $\chi^{2}=51.80, d f=24, p<.01$, Week 3 task self-efficacy $\mathrm{R}^{2}=$ .736 , Week 3 SWB $\mathrm{R}^{2}=.48$, and Week 3 physical activity $\mathrm{R}^{2}=.509$. Other indices of model fit were mixed, $\mathrm{CMIN} / d f=2.16, \mathrm{CFI}=0.92, \mathrm{RMSEA}=0.14$. These indices only partially supported a well-fitting model, as the CMIN/df statistic was the only value that indicated a wellfitting model. The CFI and RMSEA values represented a poor-fitting model. The model fit is a noted limitation. Figure 4 shows the cross-lagged paths that were tested. The cross-lagged path from Week 0 task self-efficacy to Week 1 physical activity was non-significant, $\mathrm{b}=-.660, p=$ .163. The cross-lagged path from Week 0 task self-efficacy to Week 3 physical activity was also non-significant, $\mathrm{b}=.424, p=.11$. In order to examine whether physical activity was associated with task self-efficacy instead, a path was tested from Week 2 physical activity to Week 3 task self-efficacy. This path was also found to be non-significant, $\mathrm{b}=.003, p=.88$.

Cross-lagged paths were also tested from physical activity to subsequent times of SWB. The path from Week 0 physical activity to Week $1 \mathrm{SWB}$ was non-significant, $\mathrm{b}=-.01, p=.317$, the path from Week 1 physical activity to Week $2 \mathrm{SWB}$ was non-significant, $\mathrm{b}=.005, p=.398$, and the path from Week 2 physical activity to Week 3 SWB was also non-significant, $\mathrm{b}=.012, p$ $=.267$. The cross-lagged path from Week 0 task physical activity to Week 3 SWB was non- 
significant, $\mathrm{b}=-.01, p=.63$. As none of these proposed paths were significant, additional crosslagged paths were tested to check for associations stemming from SWB to subsequent physical activity. The path from Week 0 SWB to Week 1 physical activity was non-significant, $b=-.236$, $p=.867$, the path from Week 1 SWB to Week 2 physical activity was non-significant, $\mathrm{b}=1.682$, $p=.152$, and the path from Week 2 SWB to Week 3 physical activity was also non-significant, $\mathrm{b}$ $=-1.423, p=.114$. To test for further associations, a path was tested from Week 0 task selfefficacy to Week $1 \mathrm{SWB}$, which was non-significant, $\mathrm{b}=-.03, p=.112$.

None of the cross-lagged paths were found to be significant. These findings suggest a lack of association across task self-efficacy, physical activity, and SWB. However, the autoregressive paths were significant, indicating a change within the variable over time, as was also verified by the repeated measures ANOVA analyses and paired t-test.

\section{Discussion}

The purpose of this study was to test the associations of task self-efficacy, physical activity, and SWB across 4 times of measurement, along with studying the effects of age, sex, education, and race on these associations. Previous literature has found significant associations between task self-efficacy and physical activity (Seghers et al., 2014), as well as between physical activity and SWB (Garatachea et al., 2009; McAuley et al., 2006), but the literature linking all of these aspects together, as well as information about the direction of effects is lacking. Although one of this study's aims was to address these gaps in the literature, the results do not fully support the previous literature.

\section{Hypothesis 1: Autoregressive Paths across Task Self-Efficacy, Physical Activity and SWB}

Task self-efficacy, physical activity, and SWB were all found to be associated with 
subsequent times of measurement within their measure, except for the two middle time points for physical activity. This represents a relation in scores across the various waves. Furthermore, participants were scoring the lowest in SWB, task self-efficacy and physical activity at Week 0 , but increased over the course of the study. This finding connects to the principle of health coaching, which focuses on working to increase the health and wellness of participants over the duration of the coaching (Butterworth et al, 2007). The results indicate that the health coaching was potentially beneficial in the beginning of the 3 weeks, but there were not significant changes seen across the mean scores of SWB and physical activity during the rest of the study, indicating that hypothesis 1 is only partially supported. However, the inclusion of a control group would be necessary to analyze the potential additional benefits provided by health coaching over time. Considering the pattern found from the results, potentially participants increased in their physical activity as much as possible in the first week and could not increase anymore in subsequent weeks. Another potential explanation for this is that significant change occurs not within 3 weeks, but over the course of a longer duration, which is preventing us from seeing any effects. Overall, the full 21 days of health coaching may have been beneficial for some while not as helpful for others.

\section{Hypothesis 2: Task Self-Efficacy and Physical Activity}

Hypothesis 2 was not supported due to the fact that task self-efficacy was not found to be significantly associated with subsequent physical activity. Previous literature has noted the lack of certainty for the direction of an effect between task self-efficacy and physical activity (Ayotte et al., 2013, McAuley et al., 2007; Mullen et al., 2012). However, neither task self-efficacy associated with subsequent physical activity nor physical activity associated with subsequent task self-efficacy produced a significant association in the model. Bandura (1982) discusses the 
importance of self-efficacy as it pertains to how hard a person will try to complete a task or reach a goal (Bandura, 1982). In regards to physical activity, those who are highly efficacious may put in more effort to reach their physical activity goal (Bandura, 1982). Bandura (1982) also discusses, however, that those who feel high efficacy towards their goal, such as physical activity, may ultimately spend less time working towards the goal due to a sense of overconfidence. Incorporating this information in with our results, potentially participants exhibited highly efficacious thoughts about their activity, but once it was time to be physically active, they had inadequate effort and preparation, resulting in a lack of physical activity engagement. Another possibility is that participants realized that being physically active was demanding in a physical sense and in regards to time, so participants may have exhibited higher task self-efficacy levels, but there engagement was affected by barriers. Additionally, participants may have been unsuccessful at gauging their level of task self-efficacy. Perhaps a participant believed they had high task self-efficacy, but once they started engaging in the physical activity, they made the realization that they were not as confident in their ability to be active. These are some potential explanations for why we saw a general lack of support for task self-efficacy influencing physical activity, as guided by SCT.

\section{Hypothesis 3: Physical Activity and SWB}

Hypothesis 3 was also not supported, as physical activity was not associated with SWB. Although there is a recognized association between SWB and physical activity by previous literature (Garatachea et al., 2009; Singh et al., 2005), both directions (i.e., SWB to physical activity and physical activity to SWB) were checked for a significant association, but there was not one present. The sample included in the study is potentially very different from other samples utilized. More clearly, participants could have received a total of $\$ 105$ from participating in the 
study, allowing for some participants to be motivated by the monetary compensation. Although participants were required to fill out a prescreen, assessing if participant's maintained a goal that they wished to work on with a health coach, there could have been other motivations behind participation, such as the monetary compensation, desire to volunteer, receive extra credit in undergraduate classes, or to appease others by working on becoming physically active.

\section{Hypothesis 4: Age}

As was hypothesized, there was no age effect detected, indicating that all adults scored similarly on measures of task self-efficacy, physical activity, and SWB. This finding is encouraging for older adults in that they can still be physically active and experience task selfefficacy, as well as experience SWB. Along with a lack of differences across age, there were also no differences found for sex, education, and race. Thus, both males and females scored similarly in these areas, as well as people of varying education levels and people of different races. Although data shows that older adults tend to be engaging in the least amount of physical activity (Schoenborn et al., 2013), potentially those incorporated into this study were more healthconscious individuals, a particular subset of older adults. Additionally, perhaps past statistics are now antiquated and older adults are beginning to follow physical activity guidelines more closely.

\section{Study Comparison}

One important aspect to consider, making our study different from those documented in the previous literature, was the study's duration. This study encompassed 3 weeks, which was not necessarily a significant amount of time in comparison to other studies. Fox et al. (2007) found slight improvements in SWB and quality of life, stemming from more intense physical activity engagement after participants had completed a 12-month study. In regards to task self- 
efficacy and the influence on physical activity, Seghers et al. (2014) documented an increase in task self-efficacy after a 12-week intervention that specifically targeted task self-efficacy, which then was associated with a decrease in amount of time spent in a sedentary behavior. The crucial aspect to consider, however, is that our study aimed to focus on habit acquisition, so focusing on the initial 3 weeks was paramount in order to assess how initial physical activity routine adoption occurs. Although change was also being assessed in our model, the change was specific to having just incorporated a new physical activity regiment into participants' lifestyles. This is imperative information and helps to depict the time sensitivity of physical activity routine adoption.

Furthermore, our study is different from others in the literature due to the fact that objective and subjective measures of physical activity were both utilized. Accelerometers have only become more popular in studies as of the early 2000s (Ortlieb et al, 2014), allowing for possible differences across results. Additionally, we had a small sample size, which potentially masked some of the effects found in the previous literature. Replication of this study with a larger sample size could allow for further analysis of task self-efficacy, physical activity, SWB and the associations among these variables, as well as in combination with covariates. This study also included a variety of measures from other domains, including cognition, spirituality, and personality. Potentially one or several of these other domains also influence physical activity, but as physical activity is a very complex behavior, it is difficult to see the associations across domains.

An additional explanation for our findings is that the task self-efficacy and physical activity measures rely on different conceptualizations of activity. The Task-Related Self-Efficacy Scale gauges continuous physical activity, but without a specific intensity. The Godin Leisure 
Time Exercise Questionnaire specifically asks about time spent engaged in vigorous, moderate, and mild exercise. Furthermore, Cronbach's alpha for the Godin Leisure Time Exercise Questionnaire had only adequate reliability in previous studies, as well as our own. As it was hypothesized that task self-efficacy would influence physical activity, then causing physical activity to influence SWB, if the physical activity measure was a poor measure, this could account for the non-significant cross-lagged paths. Regarding differences across sex in reporting physical activity engagement, we could not locate any recent articles describing sex differences for the Godin Leisure Time Exercise Questionnaire, although correlations for self-reported physical activity, in general, have been fairly similar across males and females (Bowles, FitzGerald, Morrow, Jackson, \& Blair, 2004). Connectedly, task self-efficacy was measured at only 2 time points whereas physical activity and SWB were measured at all 4 time points. If task self-efficacy had been measured at Week1 and Week 2, there may have been an association found.

Although not all of our findings replicate the results from previous literature, there are many strengths of this study. Such strengths include implementing a longitudinal study with multiple waves of data; applying task self-efficacy, physical activity, and SWB into one path analysis; utilizing a health coaching paradigm; and implementing subjective and objective measures of physical activity, which will be utilized in future studies. These many strengths provide insight into successful interventions, which target task self-efficacy, physical activity, and SWB.

When considering the results that we obtained and successful interventions, it would be intuitively beneficial to incorporate a longer (in weeks, months, or years) intervention, lasting at least 12 weeks, to ensure that effects have emerged, if there are any. As was discussed earlier, 
the findings by Fox et al. (2007) for increased wellbeing and Seghers et al. (2014) for higher task self-efficacy represent long-term interventions, lasting multiple months to an entire year. This longitudinal design is imperative due to the consistent autoregressive trends across the variables, as well as for the study of physical activity maintenance. Additionally, to properly assess stability and change of physical activity, at least 3 times of measurement should be included, as this is the minimum number of waves considered acceptable to view change (Singer \& Willett, 2003).

Future interventions should incorporate a health coaching aspect, as it appeared to be beneficial for some participants in our study, as well as in previous literature (e.g., Butterworth et al., 2007). More clearly, the effect of health coaching, or working with individuals in an attempt to increase their wellbeing (Butterworth et al., 2007), was seen to potentially have had an effect during the first week of the study, which is when the significant increases were seen from the baseline measures. Participants may have felt more dedicated to the study in the beginning, but less so over time. Connectedly, implementing a larger task self-efficacy coaching component could be beneficial to see the initial increase in task self-efficacy, which could then lead to subsequent associations with physical activity or other measures. This was a significant component in the past literature, which was then associated with changes in SWB and time spent in sedentary behaviors (Seghers et al., 2014). Along with health coaching, teaching participants various age-appropriate physical activities to utilize could potentially help engagement in physical activity outside of the laboratory setting (Fox et al, 2007; Singh et al, 2005). This act of incorporating specific activities into treatment could connect to participants continuing the activities for an extended period, too. This could be crucial, especially for younger adults, as it 
has been recommended that younger adults start an exercise routine so as to be more likely to maintain the activity (Marcus et al., 2000).

As was discussed previously, the parent study incorporated many different measures. These measures related back to different domains. This is a strength of the study and an aspect that should be applied in other studies so as to assess physical activity in relation to other dimensions, as it is such a complex behavior. Considering the many different measures, another crucial aspect is whether physical activity is being assessed subjectively or objectively. Although the physical activity data across self-report and accelerometers is fairly similar in content, when participants self-report their intensity of activity, this is when there appears to be an inaccuracy (Troiano, Berrigan, Dodd, Masse, Tilert, \& McDowell, 2008). Assessing whether participants are engaging in vigorous, moderate, or mild physical activity is a crucial component of analyzing whether physical activity guidelines are being met (e.g., Schoenborn et al., 2013). Thus, future interventions should utilize accelerometer or utilize accelerometers and self-report to continue to assess similarities and differences between the two.

\section{Limitations}

Although SWB and physical activity was assessed over the course of 4 waves, task selfefficacy was only utilized at 2 waves. This is a limitation because generally the lowest amount of waves considered acceptable to see change is 3 waves (Singer \& Willett, 2003). Potentially the smaller number of waves included for task self-efficacy negatively affected the results, blinding a potential association during the middle of the protocol. Considering the specific measures utilized, the Godin Leisure Time Exercise Questionnaire only resulted in acceptable reliability and the SWLS measure for SWB was found in previous literature to have only acceptable reliability. A large number of other measures were also included in the parent study, but were not 
incorporated in this study. Perhaps including other domains would have had an influence on the results.

Other limitations are associated with the representativeness of the included sample. The majority of participants were Caucasian and female. The sample was not the most representative, which is a noted weakness of the study. However, this allows us to know a good deal about this particular population. Additionally there were some instances when health coaches were unable to meet with their usual participants and a different health coach met with the participant for that session. Potentially this change in coach could affect the participant and the connection they have with the study.

\section{Future Directions}

Future research on this topic should focus on incorporating a representative sample so as to ensure generalizability of results. Future studies utilizing longitudinal data should also follow a strict protocol of having at least 3 waves of data for all included variables in order to fully assess associations, with a longer study duration most likely being more beneficial. Health coaching also needs to be studied in more detail so as to continue assessing its utility as a way of enhancing health, especially across longer intervention durations.

\section{Conclusion}

Task self-efficacy, SWB, and physical activity were all found to have significant autoregressive trends over the course of the study, with increasing means after Time 1. Age did not exert effects on task self-efficacy, SWB, or physical activity, highlighting the abilities of all adults to experience and partake in these different aspects. Support was not found for age effects across variables nor were there any sex, race, or education effects. Although health coaching may have been beneficial for some people, especially in the beginning of the study, it may not 
have had a significant effect on others, partially explaining these results. Future work must be completed to address these important issues. 


\section{References}

Anderson, E. S., Wojcik, J. R., Winett, R. A., \& Williams, D. M. (2006). Social-cognitive determinants of physical activity: The influence of social support, self-efficacy, outcome expectations, and self-regulation among participants in a church-based health promotion study. Health Psychology, 25(4), 510-20. doi:10.1037/0278-6133.25.4.510.

Andrews, F. M., \& Withey, S. B. (1976). Social indicators of well-being: Americans' perceptions of life quality.

Arbuckle, J. L. (2012). AMOS 21.0. Crawforville: Amos Development Corporation.

Arent, S.M., Landers, D.M., and Etnier, J.L. (2000). The effects of exercise on mood in older adults: a meta-analytic review. Aging Phys. Activity 8, 407-30.

Ayotte, B. J., Margrett, J. A., \& Patrick, J. H. (2013). Dyadic analysis of self-efficacy and perceived support: The relationship of individual and spousal characteristics with physical activity among middle-aged and young-older adults. Psychology and Aging, 28(2), 555-63. doi:10.1037/a0032454.

Bandura, A. (1977). Self-efficacy: Toward a unifying theory of behavioral change. Psychological Review, 84(2), 191.

Bandura, A. (1982). Self-efficacy mechanism in human agency. American Psychologist, 37, 122147.

Bandura, A. (1997). Self efficacy: The exercise of control. (pp. 1-604). New York, NY: W.H. Freeman and Company.

Bandura, A. (1998). Health promotion from the perspective of social cognitive theory. Psychology and Health, 13(4), 623-649.

Bandura, A. (2006). Guide for constructing self-efficacy scales. Self-efficacy beliefs of 
adolescents, 5(307-337).

Baranowski, T., Bar-Or, O., Blair, S., Corbin, C., Dowda, M., Freedson, R., ... \& Ward, D. (1997). Guidelines for school and community programs to promote lifelong physical activity among young people. Morbidity and Mortality Weekly Report, 50(RR-6), 1-36.

Barwais, F. A., Cuddihy, T. F., \& Tomson, L. M. (2013). Physical activity, sedentary behavior and total wellness changes among sedentary adults: a 4-week randomized controlled trial. Health and Quality of Life Outcomes, 11(1), 183.

Bowles, H. R., FitzGerald, S. J., Morrow, J. R., Jackson, A. W., \& Blair, S. N. (2004). Construct validity of self-reported historical physical activity. American Journal of Epidemiology, 160(3), 279-286.

Bray, S. R., \& Cowan, H. (2004). Proxy efficacy: Implications for self-efficacy and exercise intentions in cardiac rehabilitation. Rehabilitation Psychology, 49(1), 71-75. doi:10.1037/0090-5550.49.1.71.

Butterworth, S. W., Linden, A., \& McClay, W. (2007). Health coaching as an intervention in health management programs. Disease Management \& Health Outcomes, 15(5), 299-307.

Caspersen, C., Pereira, M. A., \& Curran, K. M. (2000). Changes in physical activity patterns in the United States, by sex and cross-sectional age. Medicine and Science in Sports and Exercise, 32(9), 1601-1609.

Chaddock, L., Voss, M.W., \& Kramer, A.F. (2012). Physical activity and fitness effects on cognition and brain health in children and older adults. Kinesiology Review, 1, 37-45. Codina, N., Pestana, J. V, \& Armadans, I. (2013). Physical activity (PA) among middle-aged women: Initial and current influences and patterns of participation. Journal of Women \& Aging, 25(3), 260-72. doi:10.1080/08952841.2013.791605. 
Diener, E. (2000). Subjective well-being: The science of happiness and a proposal for a national index. American Psychologist, 55(1), 34.

Diener, E. D., Emmons, R. A., Larsen, R. J., \& Griffin, S. (1985). The satisfaction with life scale. Journal of Personality Assessment, 49(1), 71-75.

Diener, E., Oishi, S., \& Lucas, R. E. (2003). Personality, culture, and subjective well-being: Emotional and cognitive evaluations of life. Annual Review of Psychology, 54(1), 403-425.

Dolan, P., Peasgood, T., \& White, M. (2008). Do we really know what makes us happy? A review of the economic literature on the factors associated with subjective wellbeing. Journal of Economic Psychology, 29(1), 94-122.

Emmons, R. A., \& McCullough, M. E. (2003). Counting blessings versus burdens: an experimental investigation of gratitude and subjective well-being in daily life. Journal of Personality and Social Psychology, 84(2), 377.

English, C., Manns, P. J., Tucak, C., \& Bernhardt, J. (2013). Physical activity and sedentary behaviors in community-dwelling stroke survivors: A systematic review. Physical Therapy.

Fox, K. R. (1999). The influence of physical activity on mental well-being. Public Health Nutrition, 2(3a), 411-418.

Fox, K. R., Stathi, A., McKenna, J., \& Davis, M. G. (2007). Physical activity and mental wellbeing in older people participating in the better ageing project. European Journal of Applied Physiology, 100(5), 591-602.

Friedenreich, C. M., \& Orenstein, M. R. (2002). Physical activity and cancer prevention: etiologic evidence and biological mechanisms. The Journal of Nutrition, 132(11), 3456S$3464 S$. 
Garatachea, N., Molinero, O., Martínez-García, R., Jiménez-Jiménez, R., González-Gallego, J., \& Márquez, S. (2009). Feelings of well being in elderly people: relationship to physical activity and physical function. Archives of Gerontology and Geriatrics, 48(3), 306-312.

Ghosh, D., \& Vogt, A. (2012). Outliers: An evaluation of methodologies. In Joint Statistical Meetings (pp. 3455-3460). San Diego, CA: American Statistical Association.

Godin, G., \& Shephard, R. J. (1997). Godin leisure-time exercise questionnaire. Medicine and Science in Sports and Exercise, 26(6), S36-38.

Gross, J. J. (2013). Emotion regulation: taking stock and moving forward. Emotion, 13(3), 359.

Luszczynska, A., Scholz, U., \& Schwarzer, R. (2005). The general self-efficacy scale:

Multicultural validation studies. The Journal of Psychology, 139(5), 439-457.

Lutter, J. M. (1994). History of women in sports. Societal issues. Clinics in Sports Medicine, 13(2), 263-279.

Marcus, B., Forsyth, L., Stone, E., Dubbert, P., McKenzie, T., Dunn, A., \& Blair, S. (2000). Physical activity behavior change: Issues in adoption and maintenance. Health Psychology, 19(1), 32-41. doi:10.1037//0278-6133.19.1(Suppl.).32

McAuley, E. (1993). Self-efficacy and the maintenance of exercise participation in older adults. Journal of Behavioral Medicine, 16(1), 103-113.

McAuley, E., Courneya, K. S., Rudolph, D. L., \& Lox, C. L. (1994). Enhancing exercise adherence in middle-aged males and females. Preventive Medicine, 23(4), 498-506.

McAuley, E., Jerome, G. J., Elavsky, S., Marquez, D. X., \& Ramsey, S. (2003). Predicting longterm maintenance of physical activity in older adults. Preventive Medicine, 37(2), 110-118. McAuley, E., Konopack, J. F., Motl, R. W., Morris, K. S., Doerksen, S. E., \& Rosengren, K. R. (2006). Physical activity and quality of life in older adults: influence of health status and 
self-efficacy. Annals of Behavioral Medicine,31(1), 99-103.

McAuley, E., Morris, K. S., Doerksen, S. E., Motl, R. W., Liang, H., White, S. M., ... \& Rosengren, K. (2007). Effects of Change in Physical Activity on Physical Function Limitations in Older Women: Mediating Roles of Physical Function Performance and SelfEfficacy. Journal of the American Geriatrics Society, 55(12), 1967-1973.

Mullen, S. P., McAuley, E., Satariano, W. A., Kealey, M., \& Prohaska, T. R. (2012). Physical activity and functional limitations in older adults: the influence of self-efficacy and functional performance. The Journals of Gerontology Series B: Psychological Sciences and Social Sciences, 67(3), 354-361.

Netz, Y., Wu, M. J., Becker, B. J., \& Tenenbaum, G. (2005). Physical activity and psychological well-being in advanced age: a meta-analysis of intervention studies. Psychology and Aging, 20(2), 272.

Okun, M. A., Karoly, P., \& Lutz, R. (2002). Clarifying the contribution of subjective norm to predicting leisure-time exercise. American Journal of Health Behavior, 26(4), 296-305.

Olander, E. K., Fletcher, H., Williams, S., Atkinson, L., Turner, A., \& French, D. P. (2013). What are the most effective techniques in changing obese individuals' physical activity selfefficacy and behaviour: A systematic review and meta-analysis. The International Journal of Behavioral Nutrition and Physical Activity, 29(10), 1-15.

Ortlieb, S., Dias, A., Gorzelniak, L., Nowak, D., Karrasch, S., Peters, A., ... \& Schul, H. (2014). Exploring patterns of accelerometry-assessed physical activity in elderly people. International Journal of Behavioral Nutrition and Physical Activity, 11, 28.

Pajares, F. (1997). Current directions in self-efficacy research. Advances in motivation and achievement, 10(149), 21-40. 
Palmer, S., Tubbs, I., \& Whybrow, A. (2003). Health coaching to facilitate the promotion of healthy behaviour and achievement of health-related goals. International Journal of Health Promotion and Education, 41(3), 91-93.

Phelan, E. A., Anderson, L. A., Lacroix, A. Z., \& Larson, E. B. (2004). Older adults' views of "successful aging"- how do they compare with researchers' definitions?. Journal of the American Geriatrics Society, 52(2), 211-216.

Rejeski, W.J. \& Mihalko, S.L. (2001). Physical activity and quality of life in older adults. Journal of Gerontology, 56A (2 Special), 23-35.

Renner, B., Spivak, Y., Kwon, S., \& Schwarzer, R. (2007). Does age make a difference? Predicting physical activity of South Koreans. Psychology and Aging, 22(3), 482.

Resnick, B., Palmer, M. H., Jenkins, L. S., \& Spellbring, A. M. (2000). Path analysis of efficacy expectations and exercise behaviour in older adults. Journal of Advanced Nursing, 31(6), $1309-1315$.

Rybash, J. M., Hoyer, W. J., \& Roodin, P. A. (1986). Adult cognition and aging. NY: Pergamon. Schoenborn, C. A., Adams, P. F., \& Peregoy, J. A. (2013). Health behaviors of adults: United States, 2008-2010. Vital and Health Statistics. Series 10, Data from the National Health Survey, (257), 1-184.

Segar, M., Jayaratne, T., Hanlon, J., \& Richardson, C. R. (2002). Fitting fitness into women's lives: effects of a gender-tailored physical activity intervention. Women's Health Issues, 12(6), 338-347.

Seghers, J., Van Hoecke, A. S., Schotte, A., Opdenacker, J., \& Boen, F. (2014). The added value of a brief self-efficacy coaching on the effectiveness of a 12 -week physical activity program. Journal of Physical Activity and Health, 11, 18-29. 
Singer, J. D., \& Willett, J. B. (2003). Applied longitudinal data analysis: Modeling change and event occurrence. New York, NY: Oxford University Press.

Singh, N. A., Stavrinos, T. M., Scarbek, Y., Galambos, G., Liber, C., \& Singh, M. A. F. (2005). A randomized controlled trial of high versus low intensity weight training versus general practitioner care for clinical depression in older adults. The Journals of Gerontology Series A: Biological Sciences and Medical Sciences, 60(6), 768-776.

Troiano, R. P., Berrigan, D., Dodd, K. W., Masse, L. C., Tilert, T., \& McDowell, M. (2008).

Physical activity in the United States measured by accelerometer. Medicine and Science in Sports and Exercise, 40(1), 181.

Verhoef, M., Love, E. J., \& Rose, S. A. (1993). Women's social roles and their exercise participation. Women \& Health, 19(4), 15-29.

Withall, J., Stathi, A., Davis, M., Coulson, J., Thompson, J. L., \& Fox, K. R. (2014). Objective Indicators of Physical Activity and Sedentary Time and Associations with Subjective WellBeing in Adults Aged 70 and Over. International Journal of Environmental Research and Public Health, 11(1), 643-656. 
Table 1

Means and Standard Deviations on the Measures Task Self-Efficacy, Physical Activity, and Subjective Wellbeing

\begin{tabular}{lccc}
\hline Measure & $n$ & $M$ & $S D$ \\
\hline Week 0 & 58 & 84.28 & 18.25 \\
Task Self-Efficacy & 58 & 50.24 & 36.4 \\
Physical Activity & 57 & 24.82 & 6.02 \\
Subjective Wellbeing & & & \\
Week 1 & - & - & - \\
Task Self-Efficacy & 57 & 92.71 & 65.98 \\
Physical Activity & 58 & 25.76 & 5.9 \\
Subjective Wellbeing & & & \\
Week 2 & - & - & 56.44 \\
Task Self-Efficacy & 55 & 93.69 & 5.47 \\
Physical Activity & 54 & 27.17 & \\
Subjective Wellbeing & & & 5.91 \\
Week 3 & 57 & 97.42 & 5.95 \\
Task Self-Efficacy & 56 & 27.09 & \\
Physical Activity & 57 & & \\
Subjective Wellbeing & & & \\
\hline
\end{tabular}


Table 2

Pearson's Bivariate Correlations for Task Self-Efficacy, Physical Activity, SWB, and covariates

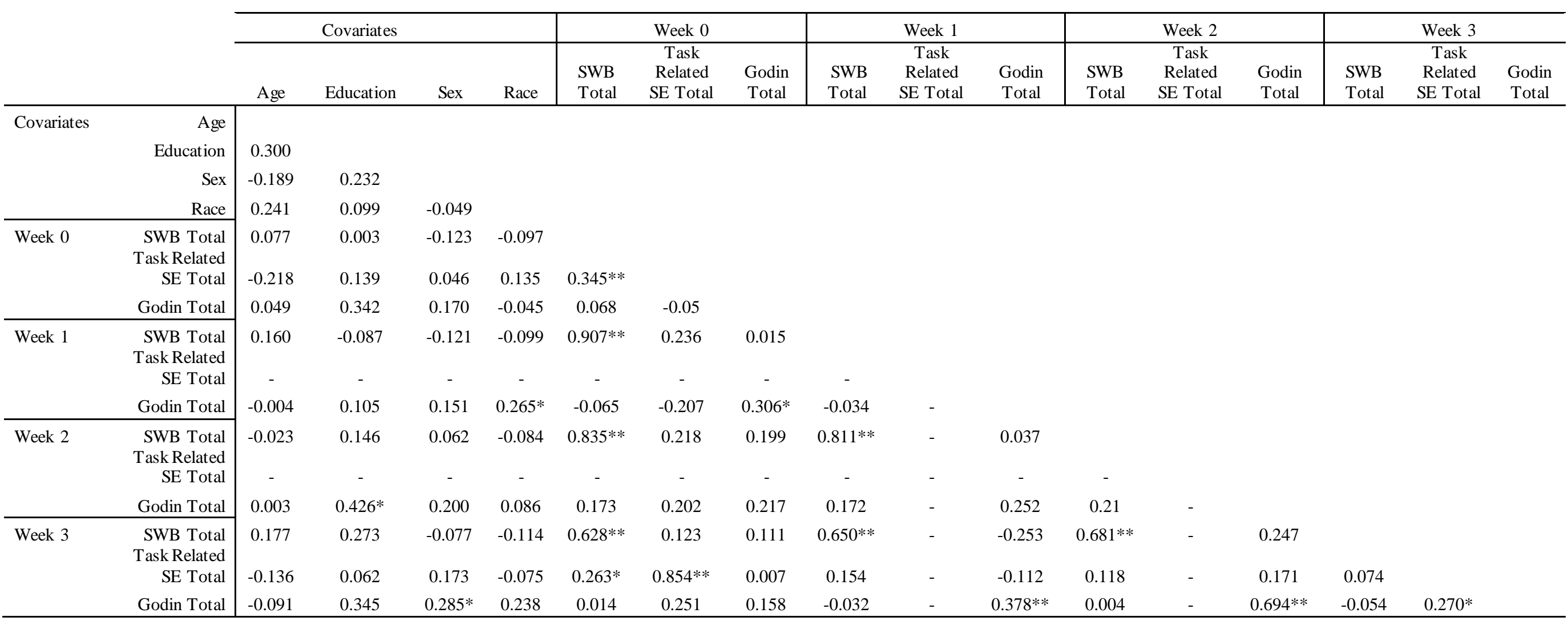

Note. "SE" is the abbreviation for self-efficacy and "SWB" is the abbreviation for subjective wellbeing. Significant values are denoted

with a "**" and is as follows: $*=p<.05, * *=p<.01, * * *=p<.001$. 
Table 3

Repeated Measures ANOVAs with Sphericity Assumed Correction for Subjective Wellbeing and Physical Activity

\begin{tabular}{lccc}
\hline Measure & $F$ & $p$ & $\eta^{2}$ \\
\hline Subjective Wellbeing & 5.84 & 0.001 & 0.10 \\
Time*Age & 1.30 & 0.278 & 0.03 \\
Time*Sex & 2.31 & 0.079 & 0.04 \\
Time*Education & 1.40 & 0.251 & 0.07 \\
Tim*Race & 0.30 & 0.829 & 0.01 \\
Physical Activity & 10.79 & 0.000 & 0.17 \\
Time*Age & 0.18 & 0.004 & 0.91 \\
Time*Sex & 0.03 & 0.995 & 0.001 \\
Time*Education & 0.41 & 0.745 & 0.02 \\
Time*Race & 1.67 & 0.176 & 0.03 \\
\hline
\end{tabular}




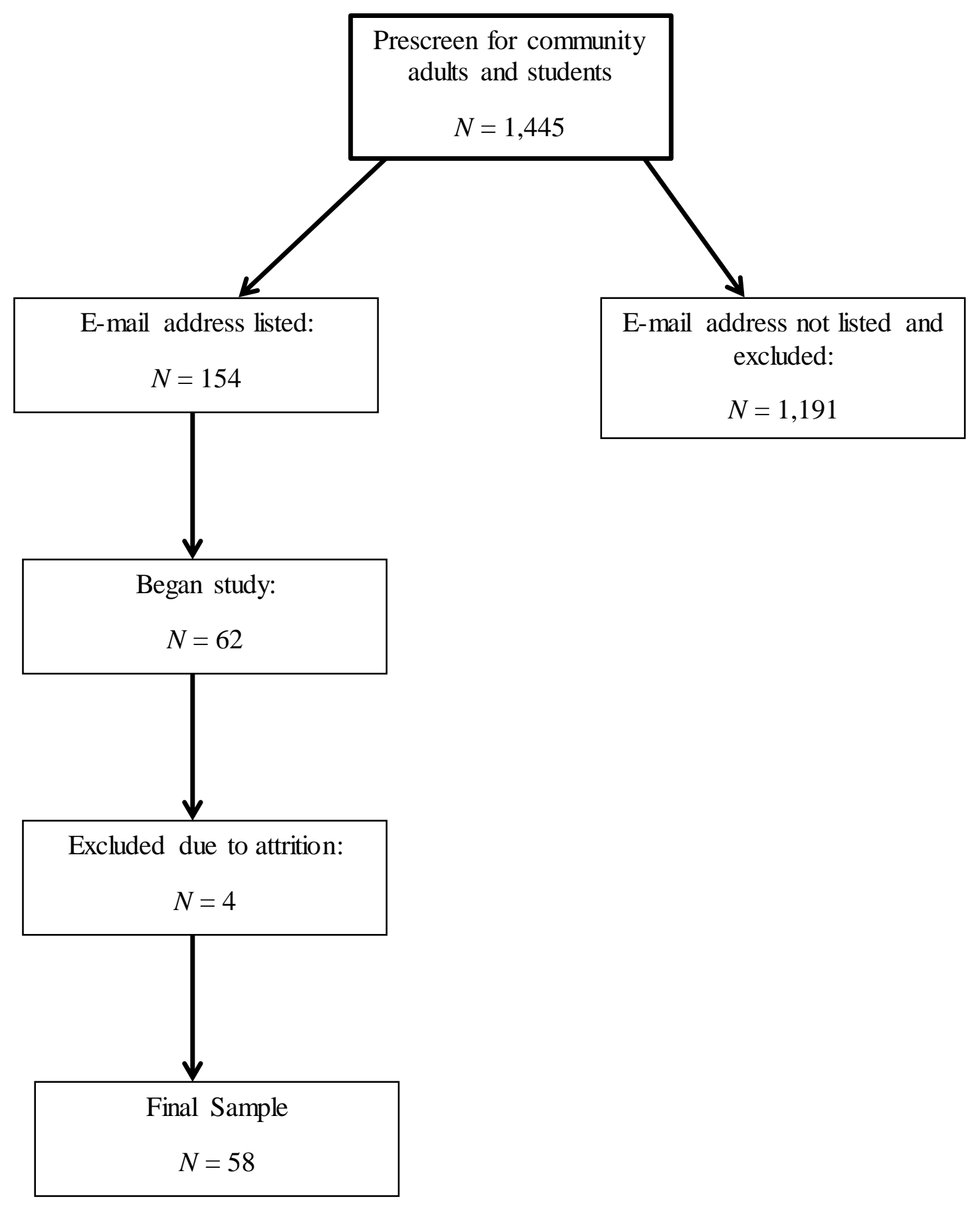

Figure 1. Flowchart detailing the process of inclusion into the study. 


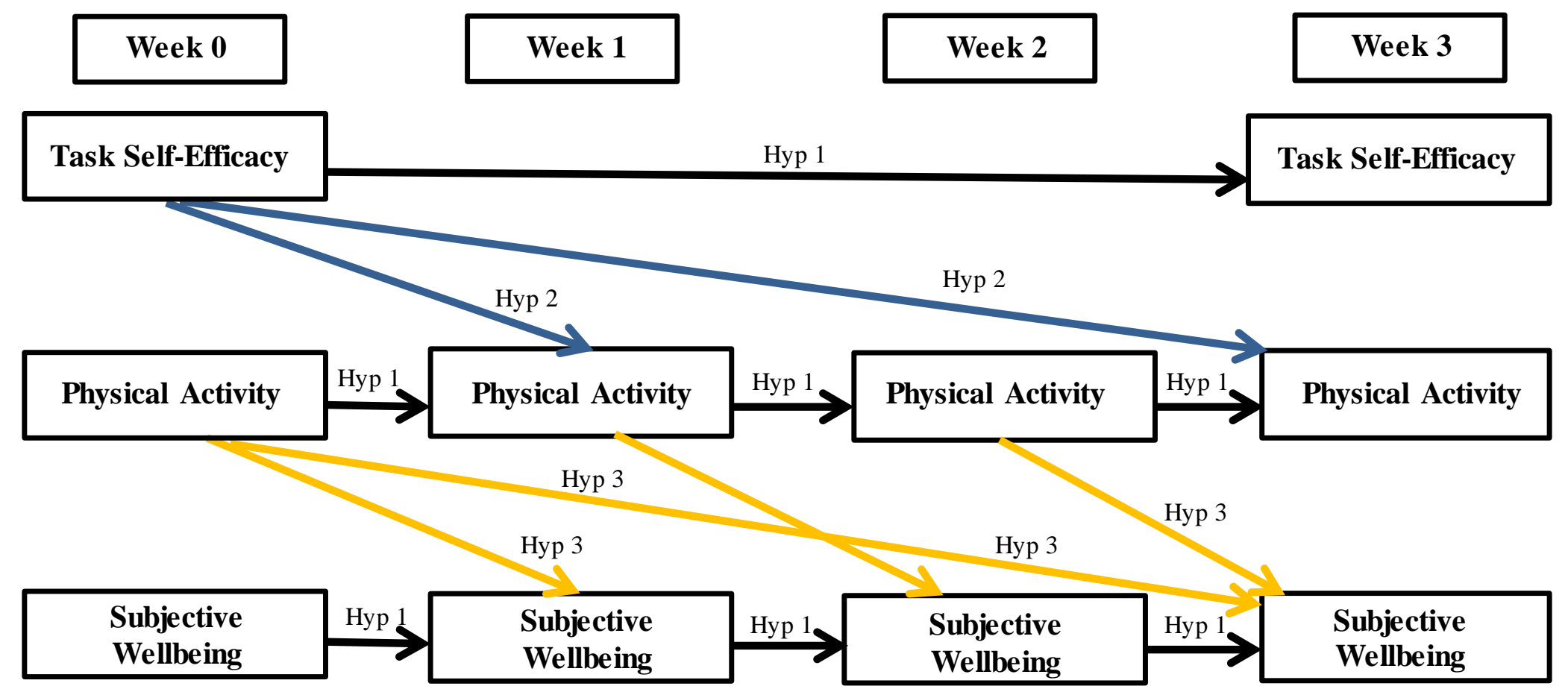

Figure 2. The black arrows represent H1, indicating a week effect for all three variables, such that there are associations between waves for each variable. $\mathrm{H} 2$ is depicted by the blue lines, indicating that initial task self-efficacy is associated with the following week's physical activity level, with a higher task self-efficacy being indicative of a higher physical activity level. The yellow arrows depict $\mathrm{H} 3$, emphasizing that the physical activity of participants directly influences the subjective wellbeing of participants, with a higher task self-efficacy being indicative of a higher physical activity level, which is then indicative of a higher subjective wellbeing. 


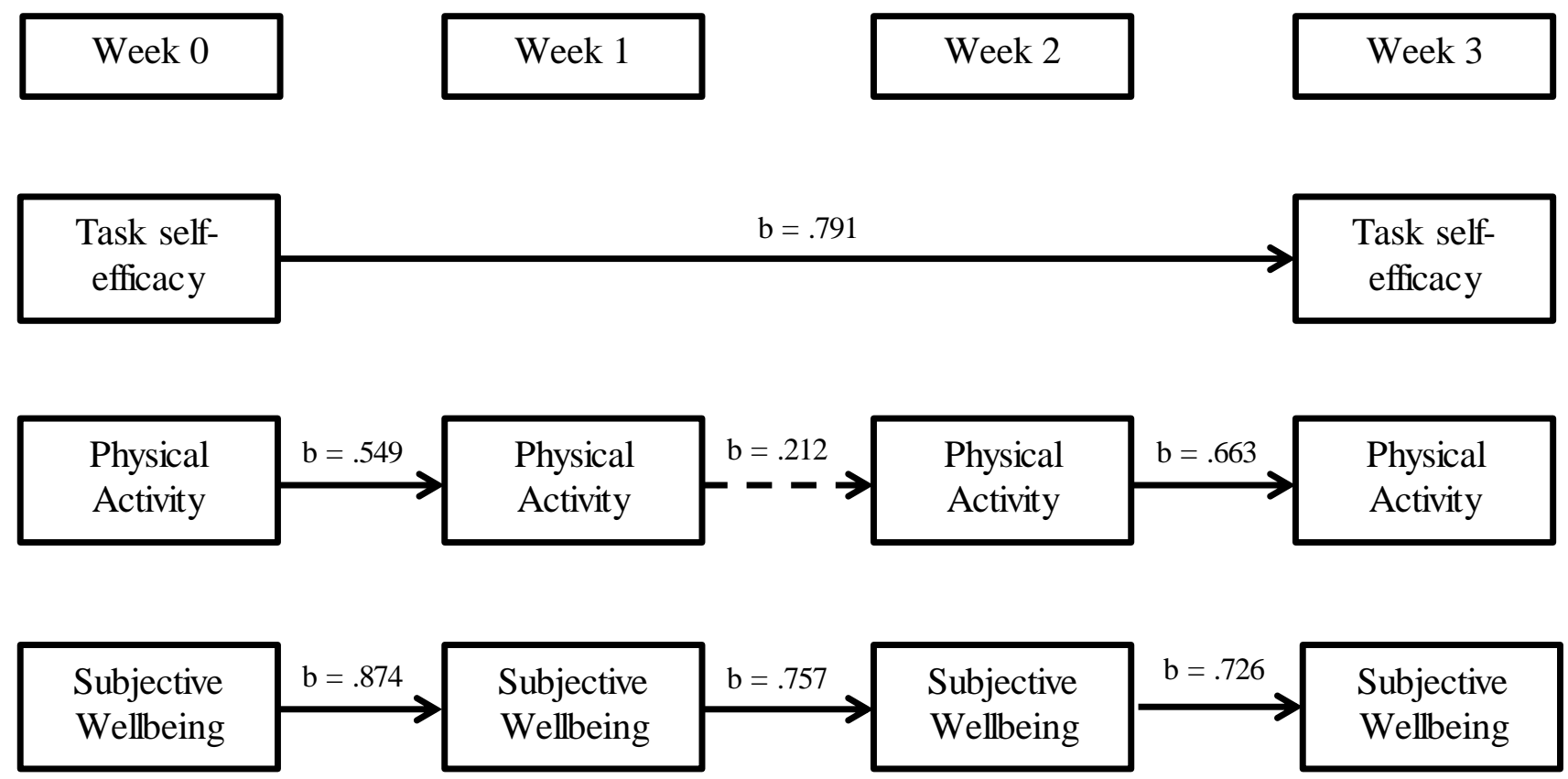

Figure 3. Autoregressive paths charted with unstandardized beta values. Solid lines indicate significant paths. Dashed lines indicate non-significant paths. 


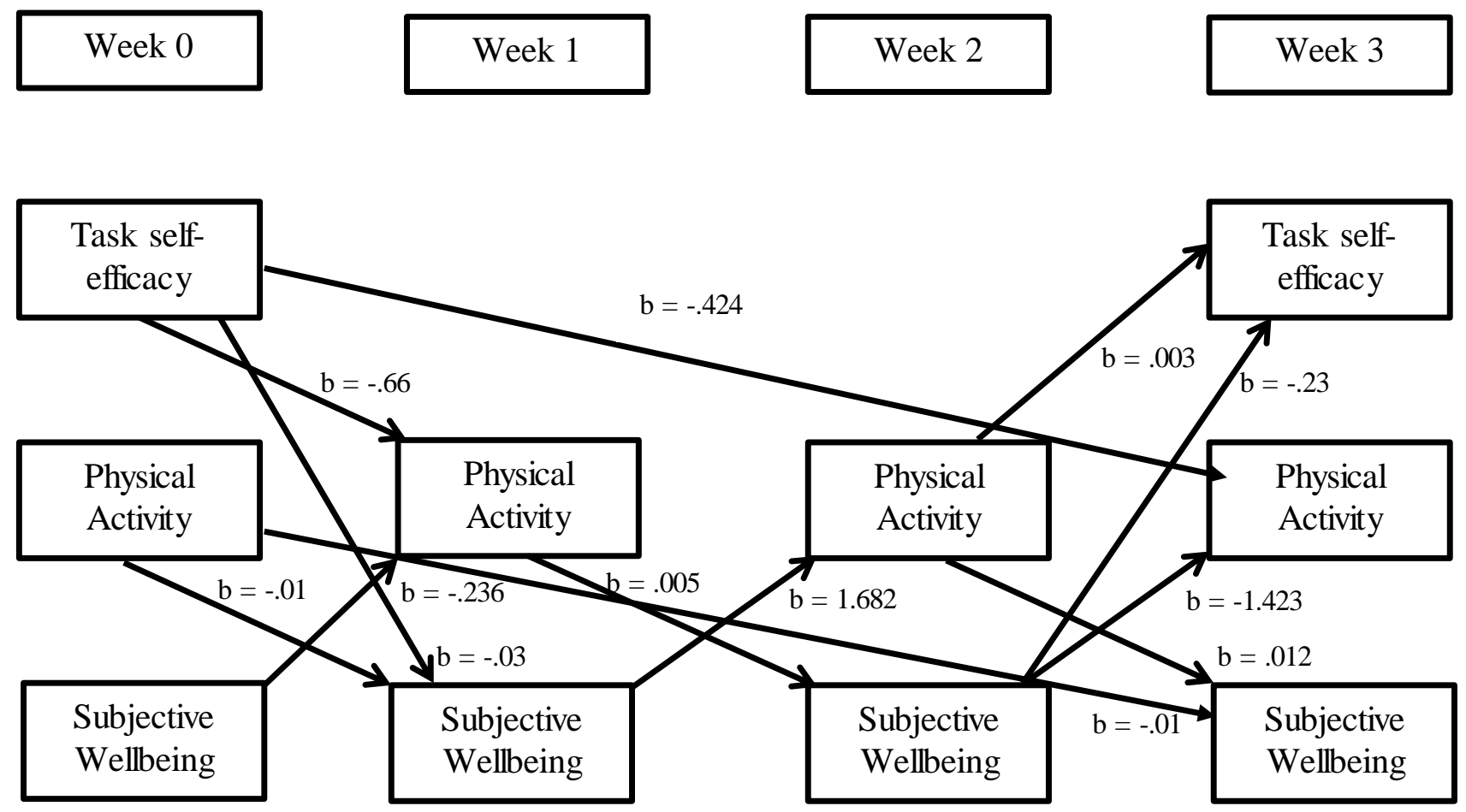

Figure 4. Cross-lagged paths with unstandardized regression weights values. 


\section{Appendix A}

The Task-Related Self-Efficacy Scale (Bray \& Cowan, 2004)

1. On a scale of $0 \%$ to $100 \%$, how confident are you that you could continuously exercise for 5 minutes?

2. On a scale of $0 \%$ to $100 \%$, how confident are you that you could continuously exercise for 10 minutes?

3. On a scale of $0 \%$ to $100 \%$, how confident are you that you could continuously exercise for 15 minutes?

4. On a scale of $0 \%$ to $100 \%$, how confident are you that you could continuously exercise for 20 minutes?

5. On a scale of $0 \%$ to $100 \%$, how confident are you that you could continuously exercise for 25 minutes?

6. On a scale of $0 \%$ to $100 \%$, how confident are you that you could continuously exercise for 30 minutes?

7. On a scale of $0 \%$ to $100 \%$, how confident are you that you could continuously exercise for 35 minutes?

8. On a scale of $0 \%$ to $100 \%$, how confident are you that you could continuously exercise for 40 minutes?

9. On a scale of $0 \%$ to $100 \%$, how confident are you that you could continuously exercise for 45 minutes? 


\section{Appendix B}

The Satisfaction with Life Scale (Diener et al., 1985)

$$
\begin{aligned}
& 7 \text { - Strongly agree } \\
& 6 \text { - Agree } \\
& 5 \text { - Slightly agree } \\
& 4 \text { - Neither agree nor disagree } \\
& 3 \text { - Slightly disagree } \\
& 2 \text { - Disagree } \\
& 1 \text { - Strongly disagree }
\end{aligned}
$$

1. In most ways my life is close to my ideal.

2. The conditions of my life are excellent.

3. I am satisfied with my life.

4. So far I have gotten the important things I want in life.

5. If I could live my life over, I would change almost nothing. 


\section{Appendix C}

The Godin Leisure Time Exercise Questionnaire (Godin \& Shephard, 1997)

During a typical 7-Day period (a week), how many times on the average do you do the following kinds of exercise for more than 15 minutes during your free time:

1. Strenuous Exercise (heart beats rapidly) (e.g., running, jogging, hockey, football, soccer, squash, basketball, cross country skïng, judo, roller skating, vigorous swimming, vigorous long distance bicycling)

2. Moderate Exercise (not exhausting) (e.g., fast walking, baseball, tennis, easy bicycling, volleyball, badminton, easy swimming, alpine skïng, popular and folk dancing)

3. Mild Exercise (minimal effort) (e.g., yoga, archery, fishing from river bank, bowling, horseshoes, golf, snow-mobiling, easy walking) 


\section{Appendix D}

\section{ABCD Prescreen and Introduction to Study}

Part 1: Participants first completed an online prescreen in order to be considered for participation in the study. The prescreen contained measures of various domains. Prompts in the prescreen directly asked potential participants if they had short-term goals that they wished to work on, if they would be interested in working with a health coach on these goals, and to leave their e-mail address if they wished to be contacted about participating in the three-week protocol. Part 2: If participants answered with the intent of wishing to work with a health coach on their short-term goals and they left their e-mail address, the PI of the study later e-mailed them, introducing them to their health coach.

Part 3: The health coach assigned to each participant then scheduled the first meeting, starting the three-week protocol.

Note: Even if those filling out the prescreen answered that they were interested, but they did not leave an e-mail address, then they were not contacted for the next portion of the study. 